\title{
Determination of electron-nucleus collision geometry with forward neutrons
}

\author{
L. Zheng ${ }^{1,2}$, E.C. Aschenauer ${ }^{2}$, and J.H. Lee ${ }^{2}$ \\ 1 Key Laboratory of Quark and Lepton Physics (MOE) and Institute of Particle Physics, Central China Normal University, \\ Wuhan 430079, China \\ 2 Physics Department, Brookhaven National Laboratory, Upton, NY 11973, U.S.A.
}

\begin{abstract}
There are a large number of physics programs one can explore in electron-nucleus collisions at a future electron-ion collider. Collision geometry is very important in these studies, while the measurement for an event-by-event geometric control is rarely discussed in the prior deep inelastic scattering experiments off a nucleus. This paper seeks to provide some detailed studies on the potential of tagging collision geometries through forward neutron multiplicity measurements with a zero degree calorimeter. This type of geometry handle, if achieved, can be extremely beneficial in constraining nuclear effects for the electron-nucleus program at an electron-ion collider.
\end{abstract}

PACS. 24.85.+p Quarks, gluons, and QCD in nuclear reactions - 29.40.Vj Calorimeters - 25.30.-c Leptoninduced reactions

\section{Introduction}

Electron-nucleus $(e+\mathrm{A})$ collisions at an electron-ion collider (EIC) provide an excellent tool for studies to understand the nuclear structure in quantum chromodynamics (QCD) with its wide kinematic reach and the ability to accelerate a large variety of nuclear beams 11. A wide range of nuclear effects can be investigated with the EIC facility. For instance, the parton distributions, especially for gluons at small momentum fraction $x$, are assumed to be largely modified by the nuclear environment and are still unconstrained. Due to the overlap of the gluon cloud from different nucleons, the gluon saturation effects may arise and are thought to be amplified with a nuclear target. At an EIC, with the precise control of $Q^{2}$ and $x$ possible by measuring the scattered electrons, this nuclear enhanced saturation effect can be systematically pinned down with measurements such as the longitudinal strucutre function $F_{L} \quad 2$ and dihadron correlations 3. Other than gluon saturation in the initial state, the nuclear medium will introduce a modification to the final state color neutralization and hadronization. The multiplicity ratio measurement $R_{A}^{h}$ can be used to examine the time development of hadronization [4.

All these effects are expected to have a strong dependence on the underlying collision geometries with respect to the nuclear environment. Gluon saturation is closely related to the impact parameter through the thickness of nuclear medium. Changes to the hadronization also correlate with the path length of fast-moving color charges in the cold nuclear medium. However, there has been little discussion about the characterization of collision geometry in individual nuclear deep inelastic scattering (DIS) collisions up to now.

In order to characterize the geometry of collisions in proton-nucleus or nucleus-nucleus collisions, quantities like the number of binary collisions $N_{\text {coll }}$, the number of nucleons participating in binary interactions $N_{\text {part }}$ and impact parameter have been extensively studied with produced particle multiplicities near central rapidity [5]. This method has been widely used in the determination of geometries for numerous measurements at the Relativistic Heavy Ion Collider (RHIC) and the Large Hadron Collider (LHC) [6,7,8]. Unfortunately, in nuclear DIS studies at the moment, geometric dependence can only be studied with the variation of nuclear target atomic number $A$, after averaging over the geometric effect for that given nuclear type.

In this work, we detail a description of the determination of collision geometry for $e+\mathrm{A}$ using the neutrons emitted at forward rapidities by target remnant evaporation process. A similar technique has been used for correcting auto-bias correlations in centrality determination of $d+\mathrm{Au}$ and $p+\mathrm{Pb}$ collisions at RHIC and the LHC 9, 10. We expound the design of an $e+\mathrm{A}$ collision geometry measurement based on simulations from the DPMJET Monte Carlo (MC) generator 11. Possible applications of this measurement have been explored. This type of geometry control, if applied to the observables in $e+\mathrm{A}$ program, not only provides an additional handle on nuclear effects 
but also simplifies the procedure of estimating systematic errors. Compared to the method of scanning multiple nuclear types, one only has to deal with the same systematic uncertainties in one nuclear type instead of worrying about several systematic uncertainties from different nuclear beams.

The remainder of the paper is organized as follows: in the Sec. 2, we introduce the relevant quantities utilized to describe the collision geometry and illustrate our strategy for categorizing different geometries. The results of this categorization are provided in Sec. 3. Possible applications of this measurement are developed in Sec. 4. In the end, we summarize our methods in Sec. 5 .

\section{Characterization method of collision geometry}

It has been argued that soft neutron production in leptonnucleus collisions is a sensitive probe of final-state interactions in the nuclear environment [12,13. To our current understanding, such neutrons are produced in the thermal emission stage of the residual nucleus left after interactions between the fast probe and target. In the first approximation, the soft neutron production is proportional to the number of nucleons removed in the DIS interaction and the subsequent secondary interactions between the particles generated in DIS process and the rest of the nucleons. In the following discussions, it is preferable to use the target rest frame with the virtual photon defining the longitudinal direction.

Conventionally, the procedure of an electron scattering from nucleus can be described by the following steps. With the electromagnetic exchange, the incoming electron emits a photon, which couples with the nucleus target. Depending on kinematics, the photon projectile then goes through one or multiple collisions with partons from the nucleons sitting in the photon's path, which can be interpreted in various frameworks [14. Here, we define the length of the projected straight trajectory of the incoming virtual photon through the nucleus, starting from the involved nucleon during the DIS process by the traveling length $d$ in the nuclear rest frame, see Fig. 1. If there are multiple nucleons involved in the DIS scattering, $d$ is defined with an interaction point from the average position of all involved nucleons. Therefore, in each event, one has the position of the involved nucleon (or average position of multiple nucleons) as the photon-nucleon primary collision vertex, as presented in Fig. 1, at a displacement of $R$ from the center of the nucleus with an impact parameter $b$.

For large nuclei, a Woods-Saxon distribution is often used to describe the initial nuclear density [15]:

$$
\rho(R)=\frac{\rho_{0}}{1+e^{\frac{R-R_{0}}{a}}},
$$

in which $R_{0}$ corresponds to the typical nuclear radius $\rho_{0}$ is the nucleon density in the center of the nucleus and $a$ gives the skin depth.

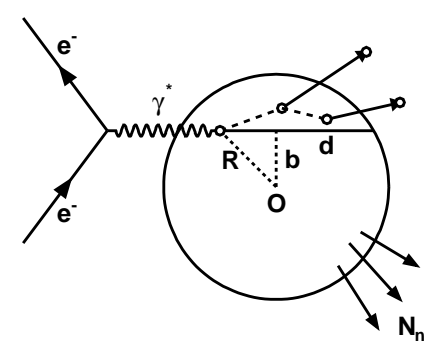

Fig. 1. Relevant quantities to describe the collision geometry. $b$ represents the impact parameter. $R$ shows the spatial displacement of the interaction point to the center of the nucleus. $d$ is the traveling length, which defines the projected virtual photon traveling length from the interaction point to the edge of the nuclear medium.

The hadronic fragments generated from photon-nucleon collisions may cause sequential secondary interactions that knock out additional nucleons not involved in the DIS interactions. This process is usually named as the "intranuclear cascade" process [16.

After all the formed final-state particles leave the nucleus remnant, due to momentum conservation, a recoil momentum will lead the residual nucleus to an equilibrium state characterized by its mass, charge and excitation energy. At the end of the reaction chain, the excited nucleus will break up into stable final products, with the emission probability described by the nuclear evaporation model [17.

In the statistical evaporation model, the number of neutron emissions strongly depends on the excitation energy, which comes from the number of nucleons removed from the nuclear remnant, dictated by the successive primary and secondary interactions in the cascade process [18]. One may find the connection between the collision geometry and the evaporated neutron number distribution through the traveling length $d$ defined above. The larger $d$ is, the more nucleons are expected to be involved in the sequential collisions and removed from the nuclear remnant, and the more neutrons can be emitted during the evaporation.

Given such a connection, one may propose that if the emitted neutron numbers can be measured, we would have a handle to effectively constrain the underlying collision geometries, which is missing for a long time in the nuclear DIS studies where the averaged geometry over the whole nucleus has typically been used. The number of neutrons emitted in the nuclear break up process will be labeled as $N_{n}$, illustrated in Fig. 1.

Once one can select for traveling length, impact parameter (centrality) can be effectively constrained according to the traveling length. As is shown in Fig. 2, events with very central collision $(b \approx 0)$ can be acquired with 
the selection of the largest traveling length (blue region), although we may have little control on the peripheral collision events; the red region corresponds to small traveling length, but it is mixed with central and peripheral collisions.

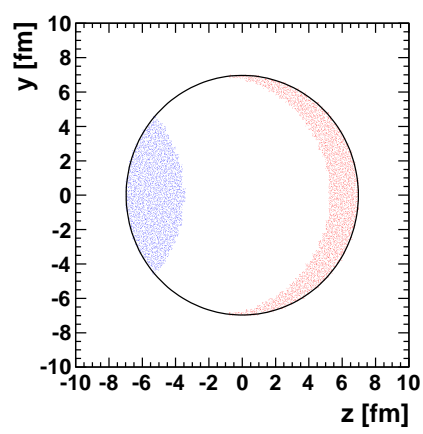

Fig. 2. A Profile view of the nucleus in the $y-z$ plane in the nuclear rest frame is presented. $y$ shows the impact parameter direction with the virtual photon going in the positive $z$ direction. The radius of the nucleus has been set to $R_{0}=6.52 \mathrm{fm}$ for gold. The regions showing the position of nucleons involved in the DIS interaction selected by $d<0.25 R_{0}$ and $d>1.5 R_{0}$ are marked by red and blue, respectively.

The Monte Carlo generator DPMJET has been utilized in our studies to simulate $e+\mathrm{A}$ collisions. In the Monte Carlo procedure, initial nuclear geometric configurations are built with nucleons drawn randomly with a radius $R$ to the center of target ion from the distribution $4 \pi R^{2} \rho(R)$ where $\rho(R)$ follows the Woods-Saxon distribution. For the simulation of a gold nucleus, we have $R_{0}=1.12 A^{1 / 3}=6.52 \mathrm{fm}$ and skin depth $a=0.545$ fm 19 .

The final states in DPMJET are simulated by three stages in a chain. Firstly, the primary DIS interactions are simulated by PHOJET 20. Only primary DIS interactions can generate particles with a hard momentum transfer. Then, particles produced at the primary interaction become the source to trigger the intranuclear cascade process. A formation zone concept has been introduced to this cascade process. In the target rest frame of the DIS interaction, a formation time $\tau$ is needed before newly created particles can re-interact with the spectator nucleons [18:

$$
\tau=\tau_{0} \frac{E}{m} \frac{m^{2}}{m^{2}+p_{\perp}^{2}},
$$

with $\tau_{0}$ being a free formation length parameter. $E, m$ and $p_{\perp}$ are the energy, mass and transverse momentum for the created particles respectively. For each hadron, a formation time is sampled from an exponential distribution with an average value as given above. The lower the hadron energy is, the higher is the probability for that hadron to form inside the nucleus. The kinematics of the secondary interactions occurring in the cascade are treated by HADRIN 21. Since the nuclear remnant undergoes equilibration before breakup, evaporative particles should follow a thermal distribution. Details of the evaporation process are handled by FLUKA 22 with the input of remnant charge, mass and excitation energy and no memory from the prior stages.

It should be noticed that this formation zone intranuclear cascade model is only one way to describe the effect of final-state interactions in the nuclear environment. Many other models have more sophisticated considerations with the prehadron stage or parton energy loss incorporated. Phenomenological studies have been done by adjusting the prehadron formation time and the final physical hadron formation time to obtain the best description to the experimental data 23 . Other QCD-inspired models, which are focused on the struck quark energy loss, calculate the modifications to the fragmentation function through gluon bremsstrahlung in a nuclear medium 24 , 25. No hadron re-interaction is included in this type of model, as the produced hadron is assumed to always form outside the nucleus. In this work, we are mainly interested in the correlation between the underlying geometry and the neutron emission during evaporation. Although they are bridged through this final state interaction in the nucleus, the exact details are not very important to our study. We are aware of the possibility to apply other models like GEMINI [26], SMM [27] to treat the nuclear break up process. But as DPMJET is currently the one most widely used, we concentrate in this paper to illustrate the potential of the measurement on DPMJET only. More well-developed models, with better descriptions to the final state interaction, can be added to our discussions in the future.

Multiple scatterings have been implemented via the Gribov-Glauber realization 28 in this MC model. The primary interaction is sampled from a sequence of independent binary photon-nucleon collisions based on an elementary photon-nucleon cross section. When we have more than one nucleon coupled to the DIS interaction stage, the primary interaction point will be defined as the average position of all the involved nucleons. However, considering the elementary cross section is very small, the number of binary collisions happened in one event is most likely to be one. Coherent scattering effects have been incorporated as a shadowing of the elementary cross section, based on the coherent length of the photon probe hadronic fluctuation [29].

\section{Collision geometry categorization}

\subsection{Geometry constraint with forward neutrons}

In the following discussions, we will use the conventional variables to describe the kinematics in a DIS event. The variable $x=Q^{2} / 2 M \nu$ is the Bjorken variable, with $Q^{2}$ being the square of the four-momentum of the exchanged virtual photon, and $\nu$ showing the virtual photon energy in the target rest frame, if we denote the nucleon mass as $M$. The variable $y$ shows the inelasticity of the event measuring the fraction of the electron energy taken by the 


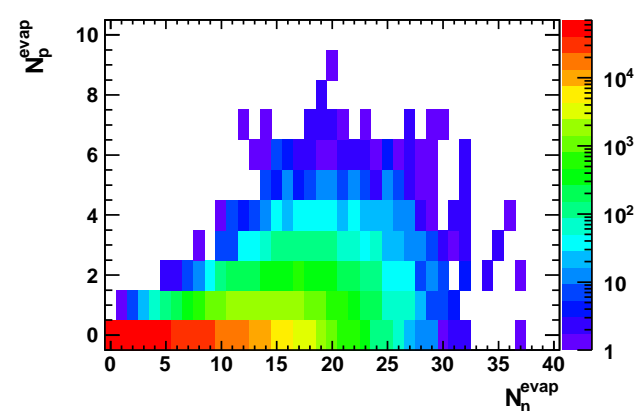

Fig. 3. Correlation between the number of evaporated protons $\left(N_{p}^{e v a p}\right)$ and neutrons $\left(N_{n}^{e v a p}\right)$. Due to the Coulomb barrier for protons, the proton emission during evaporation process is greatly suppressed compared to that of neutron.

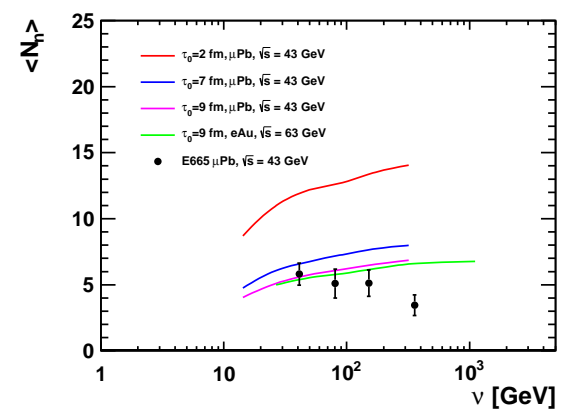

Fig. 4. Results of different formation length parameters as a function of $\nu$ in the simulation compared with the E665 data [30. The green line shows the prospective $e+\mathrm{Au}$ data kinematics coverage at an EIC.

exchanged boson. The event sample analyzed in this study is generated from DPMJET for $e+\mathrm{Au}$ at $10 \mathrm{GeV} \times 100$ $\mathrm{GeV} / \mathrm{u}$ with $1 \mathrm{GeV}^{2}<Q^{2}<20 \mathrm{GeV}^{2}, 0.01<y<0.95$.

The evaporated products emitted during nuclear break up are most likely to be protons and neutrons. Due to existence of the Coulomb barrier for charged fragments, proton emission will be largely suppressed. As seen in Fig. 3 , the number of emitted protons during nuclear evaporation is much lower than that of neutrons from the same event. Therefore, by measuring neutrons alone, we can characterize the major properties of the nuclear break up process.

The zero degree calorimeter (ZDC) designed to measure neutral energy deposits within a small radiation cone about the beam direction can be employed in the measurement of spectator neutrons emitted with a small angle from the beam remnant. Meanwhile, charged fragments and the noninteracted beam remnants will be bent to larger angles out of the ZDC acceptance by deflecting magnets. Thus, a ZDC reads out the total neutral energy in the forward rapidities, or effectively the number of emitted neutrons, from nuclear break up in a very clean way.

There's currently only limited knowledge about the magnitude of this neutron emission for DIS events, the only available measurement being from E665 experiment 30]

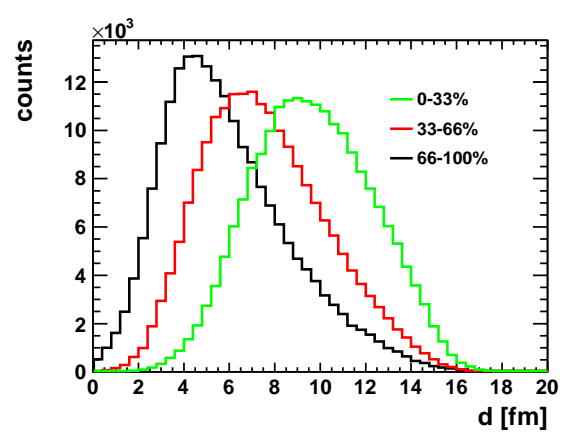

Fig. 6. Traveling length distribution in different forward neutron energy bins. The black line corresponds to peripheral collisions (66-100\%), while the red and green lines correspond to the $33-66 \%$ and $0-33 \%$, respectively.

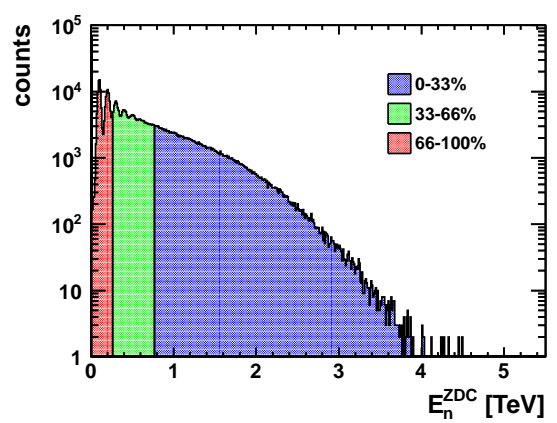

Fig. 7. Selections of different collision geometry with neutron number translated to energy deposition. Different colors represent different centrality selections

at FermiLab. From the comparison of Fig. 4 one can draw an effective formation length $\tau_{0}=9 \mathrm{fm} / c$ to consistently describe the magnitude of neutron emission. At the planned EIC energy scale, this measurement can be developed with much better precision and even wider kinematic range, to achieve a deeper understanding of this nuclear remnant response.

If well measured, the neutron number can be used as a handle of the collision geometry. However, since it is impossible to directly measure the number of neutrons, we will use the energy deposition in a ZDC to extract the neutron number information. In the following discussions, an energy resolution $\sigma / E=(85 / \sqrt{E}+9.1) \%$ from Ref. 33] has been used as the ZDC responses for neutron energy. The resolution of the traveling length is dominated by its intrinsic correlation with the number of emitted neutrons during the evaporation process. The assumed ZDC energy resolution, which is used in the current RHIC heavy ion experiments, is sufficient for our study. Based on the ZDC performance during RHIC running over a wide range of energies, an efficiency close to $100 \%$ to measure the forward neutrons with a negligible background has been assumed for all the studies in this paper.

Indicated by Fig. 5, energy deposition in the ZDC can be used as a good measure of traveling length $d$ while 


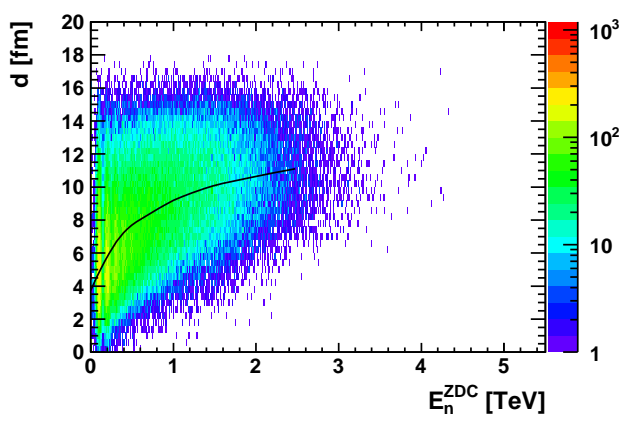

(a)

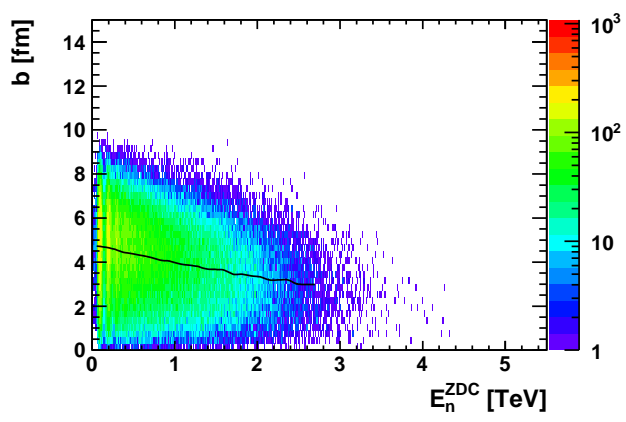

(b)

Fig. 5. (a) Scatter plot of traveling length $d$ and the energy deposition in the ZDC. The central value of $d$ in every $E_{Z D C}$ bin is indicated by the black line. (b) Scatter plot of the impact parameter $b$ and energy deposition in the ZDC. The central value of $b$ in every $E_{Z D C}$ bin is indicated by the black line.

\begin{tabular}{|l||l|l|l|}
\hline & $\Sigma E_{n}$ range $[\mathrm{GeV}]$ & $\langle d\rangle / d_{\text {RMS }}[\mathrm{fm}]$ & $\langle b\rangle / b_{\mathrm{RMS}}[\mathrm{fm}]$ \\
\hline $0-33 \%$ & $743-4329$ & $9.7 / 2.7$ & $3.8 / 1.6$ \\
\hline $33-66 \%$ & $237-743$ & $7.5 / 2.8$ & $4.4 / 1.7$ \\
\hline $66-100 \%$ & $0-237$ & $5.9 / 2.8$ & $4.7 / 1.8$ \\
\hline
\end{tabular}

Table 1. Collision geometry constrained by the selection of the neutron energy deposition in the ZDC with the method suggested in Fig. 7 . The average value as well as the root mean square (RMS) for the traveling length $d$ and the impact parameter $b$ are presented in this table.

the impact parameter $b$ is not as well controlled. Only the most central events $(b \sim 0)$ can be selected with the largest evaporated neutron emissions. With this correlation shown in Fig. 5(a), one can select a binning method to constrain the underlying geometries. Fig. 6 shows to what extent the traveling length $d$ can be constrained with the binning method shown in Fig. 7. The percentage is defined by the fraction of events with a certain energy deposition in the ZDC. Constraints put on the quantities with statistical uncertainties under the current binning strategy can be found in Tab. 1 .

The assumed polar angular acceptance of the ZDC is \pm $4 \mathrm{mrad}$ with respect to the gold nuclear beam direction [1]. Fig. 8 shows that emitted neutrons from the evaporation process can be $100 \%$ accepted. The final state neutrons from all processes are marked by the black line in that plot. The green line shows neutrons from primary interactions. Neutrons generated by intranuclear cascade are shown in blue and evaporated neutrons are shown in red. It can be concluded from this plot that neutrons from primary interactions are mainly in the midrapidity region, while the forward region neutrons are dominated by the evaporated neutrons. The primary interaction and intranuclear cascade process can also become a source for final state neutrons accepted by the ZDC. In the simulated event sample $14.66 \%$ of the accepted neutrons in the ZDC come from processes like primary and secondary interactions. As to the expected detector design, all the evaporated neutrons can be fully accepted by the experimental device and contamination from other process is under control.

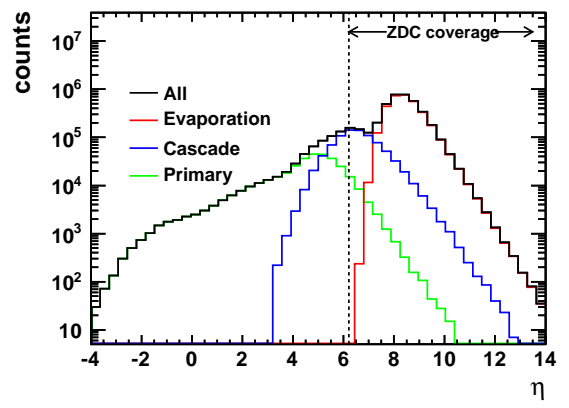

Fig. 8. $\eta$ distributions of final state neutrons from various processes. Most of the evaporated neutrons can be accepted if the ZDC covers the polar angle range of $\pm 4 \mathrm{mrad}$ marked by the dashed vertical line. Black lines represent all the final state neutrons, while the red, blue and green lines illustrate neutron distributions from evaporated, intranuclear cascade and primary productions.

\subsection{Possible constraint on the most central collisions with a double cut method}

The features of particle yield at different stages of the nuclear response are particle-type-dependent. As for the neutrons, they are mostly generated in the evaporation process from the "cooling" of a large excited nucleus. As shown in Fig. 9, the ZDC accepted neutrons are mostly evaporated neutrons. By measuring the most forward neutral energy deposition in the ZDC we can extract the statistical emission for that event in a very clean way. 


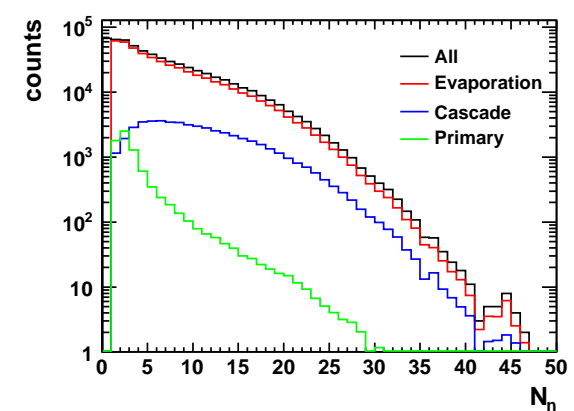

Fig. 9. Number of neutron distributions from different interaction stages. The black line shows the total number of neutrons within the ZDC acceptance; neutrons from the thermal evaporation process are depicted by the red line, while the blue line gives the distribution from secondary interactions and particles generated in primary interactions are marked in green.

With the intranuclear cascade process, additional particles like pions will be generated in the reaction chain between the fast moving particles and the rest of the nucleons. As the $p_{T}$ kick from this type of reaction is very small, charged pions generated in this process will move to the more forward direction compared to those pions from primary interactions.

With the knowledge of the underlying traveling length for the collision geometry, it is possible to define the most central collision by selecting events with the largest neutron production, in which the incoming photon probes the densest area of the target. The forward proton track number from break up and the charged particle (mostly pions) yield in forward rapidities are also sensitive to the collision geometries. We will see how much we can gain as a constraint on most central collisions if we put an additional cut on these quantities together with that on the forward neutrons. It may be beneficial to add the large neutron emission cut and the large proton or charged particle production cut at forward rapidity at the same time to select the extremely central bins. Forward proton number is measured with a perfect resolution in an assumed coverage the same as ZDC. Forward charged particles are supposed to be counted by devices covering $4<\eta<6$. The geometry constraint in the most central collisions is shown in Fig. 10. The shaded region displays the total distribution for $d$ or $b$ without any cuts. To compare with the cuts made by $0-10 \%$ selection, the magnitude of total distribution has be rescaled by 0.1 for the purpose of demonstration. Comparing the solid line and shaded region in Fig. 10 shows the forward neutron cut effectively finds the events with most central collisions. No significant enhancement can be found with the inclusion of the double cut method from forward proton or charged particles. Such ZDC accepted neutron energy would be enough to select the most central events.

\section{Applications of collision geometry constraint at an EIC}

As both initial and final state effects in $e+\mathrm{A}$ collisions are highly dependent on nuclear geometry, applying this nuclear geometry handle to our measurements in $e+\mathrm{A}$ program at an EIC will allow us to learn more about the nuclear medium effect. For instance, the selection of very central collisions maximizes the probed gluon density in the initial state which delivers more sensitivity to the expected saturation effect. $F_{L}$ and dihadron correlation measurements are two important observables sensitive to this geometry constraint. We would see stronger saturation effects in the most central collisions compared to peripheral $e+\mathrm{A}$ events with the change of gluon densities. This collision geometry also provides an additional dimension to the study of final state effects. The time space feature of this fragmentation process can be directly confronted with the nuclear medium geometry through which one can explore the hadronization process in a more precise way.

\subsection{Energy loss measurement in the cold nuclear medium}

It has been argued that the multiplicity ratio measurement $R_{A}^{h}$ from HERMES suggests that the space-time development of the hadronization process in the nuclear medium can be studied with the semi-inclusive deep-inelastic scattering process off nuclei $32,33,34$. We observe the final state hadrons with characteristic kinematic variables, such as the transverse momentum $p_{T}$ and the fractional energy of the hadron relative to the virtual photon $z$. Depending on the kinematic variables, the hadron formation may take place inside the nucleus, or outside the nucleus. In general it is assumed that the struck quark forms its full hadron identity with an average formation length $l_{h} \propto f(z) \nu$.

The multiplicity ratio $R_{A}^{h}$ is a frequently studied quantity which effectively describes the nuclear medium energy loss effect. It is defined as follows:

$$
R_{A}^{h}=\frac{\left(\frac{N_{h}\left(\nu, Q^{2}, p_{T}^{2}, z\right)}{N_{l}\left(\nu, Q^{2}\right)}\right)_{A}}{\left(\frac{N_{h}\left(\nu, Q^{2}, p_{T}^{2}, z\right)}{N_{l}\left(\nu, Q^{2}\right)}\right)_{D}},
$$

where $N_{h}$ is the semi-inclusive production of hadrons in a given kinematics bin and $N_{l}$ is the yield of leptons in the same $\nu, Q^{2}$ bin. This ratio is defined for the hadrons production per deep-inelastic scattering event on a nuclear target with mass $A$ to that of the lightest isoscalar nuclear target deuterium.

With a handle to constrain the traveling length in the collision geometry, it is possible to explore the interplay between color object neutralization and the nuclear environment geometry. If it is possible to control the traveling length $d$ of the struck quark, one has an additional dimension in this measurement which changes the measurement from $N_{h}\left(\nu, Q^{2} p_{T}^{2}, z\right)$ to $N_{h}\left(\nu, Q^{2} p_{T}^{2}, z, d\right)$. Instead of varying the different nuclear types, the hadron formation length can be directly confronted with a traveling length 


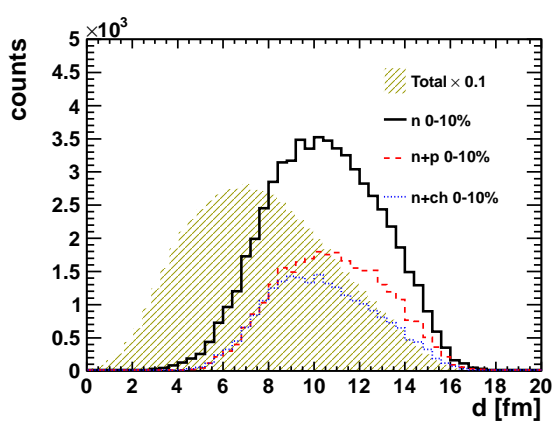

(a)

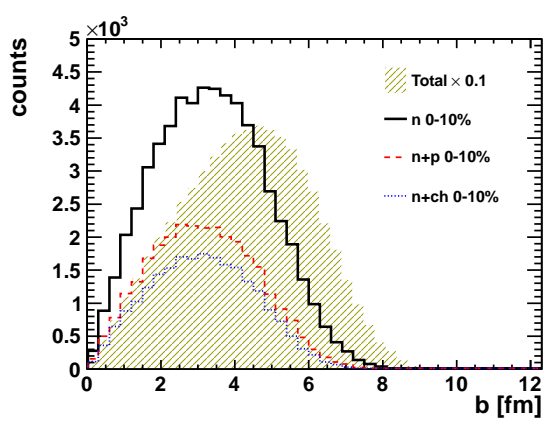

(b)

Fig. 10. Binning constraint on traveling length $d$ and impact parameter $b$ for most central collisions (0-10\%), with forward neutron cut only (solid line) and neutron cut plus additional forward proton number cut (dashed line) or forward charged hadron number cut (dotted line). The total distribution has been marked by the shaded region with a magnitude scaled down by a factor 10 for illustration.

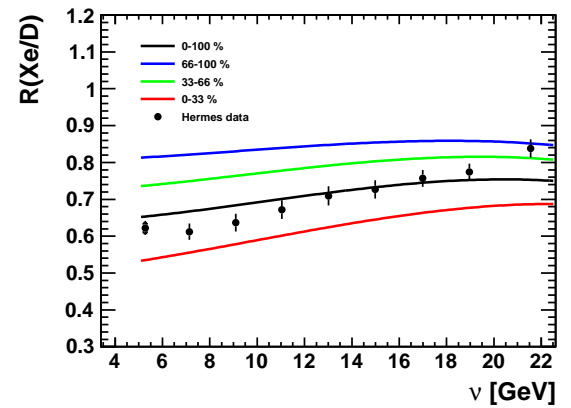

Fig. 11. Multiplicity ratio as a function of $\nu$ with the traveling length constraint. The black line represents the expected value for minimum bias events, and projected result from three centrality bins are shown in blue, green and red according to our selection discussed in Sect. 3. Hermes data points [33] are shown in the plot with black dots.

$d$; if $d>l_{h}$ it is generally formed inside the nucleus and the hadron yield will be suppressed while for $d<l_{h}$ the medium modification is supposed to be small. With the bin selection of collision geometry, we have extracted the $d$ distribution from these bins as input to an energy loss model [24]. Thereby, strong nuclear medium dependence is expected in the multiplicity ratio measurement, shown in Fig. 11.

\subsection{Dihadron correlation measurements to probe gluon saturation}

In the small $x$ limit, gluon density in the nucleon becomes so large that a phenomena named saturation may emerge. It is suggested in this scenario that, when the probe resolution $Q^{2}$ is less than the saturation scale $Q_{s}^{2}$, the gluon recombination mechanism becomes dominant, which tames the rapid growth of gluon density at small $x$. According to the collision geometry, stronger saturation is expected for the most central collisions. If this type of physics exists, evaporated neutron number can be used as an additional handle, together with the kinematics control, for studying the saturation effect.

Dihadron correlations in $e+\mathrm{A}$ are a way to investigate the saturation effect. By selecting the trigger associate particle pairs with certain $p_{T}$, one can explore the underlying jet properties and the interplay with the saturation scale $Q_{s}$. The dihadron correlation function can be constructed with the azimuthal angle difference, $\Delta \phi$, between the trigger and associate particle in the same event:

$$
C(\Delta \phi)=\frac{N_{\text {pair }}(\Delta \phi)}{N_{\text {trig }}}
$$

where $N_{\text {pair }}(\Delta \phi)$ is the number of correlated hadron pairs in every $\Delta \phi$ bin and $N_{\text {trig }}$ shows the total number of trigger particles in the event sample. The saturation scale can be parameterized as a scaling model, $Q_{s}^{2}=A^{1 / 3} c(b)\left(\frac{x}{x_{0}}\right)^{-\lambda}$, with $x_{0}=3.04^{-4}, \lambda=0.288$. $c(b)$ defines the thickness of the nuclear medium at impact parameter $b$. Significant saturation effects arise when $Q_{s}$ is large, which means collisions with smaller $x$ or larger thickness favor a stronger saturation effect.

Following the formalism in 35, one can estimate the centrality dependence in $e+\mathrm{A}$ dihadron correlations, which is coordinated by the parameter $c(b)$. From the simulation based on our centrality definition above, we can select the events with the most central and most peripheral collisions. Observable suppression is expected from peripheral to central events.

Fig. 12 shows the predicted saturation impact on dihadron correlations with an integrated luminosity of $10 \mathrm{fb}^{-1}$ at the energy of $\sqrt{s}=63 \mathrm{GeV}$. The effect of selecting the most central collisions has been studied. The thickness function is estimated by a Woods-Saxon density as in Eq. 1. For $0-10 \%$ centrality $c(b)=0.75$, while $c(b)=0.59$ for a minimum bias (0-100\%) estimation. With the current statistical uncertainty, the most central category can be slightly distinguished from the minimum bias collisions. 


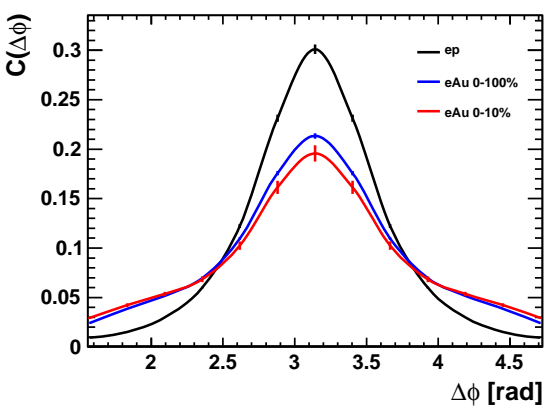

(a)

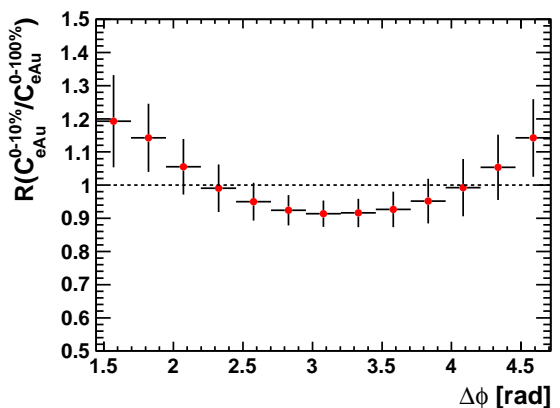

(b)

Fig. 12. Saturation effects in the dihadron correlation measurements with the most central collisions. (a) The correlation functions for $e+p, e+\mathrm{Au} 0-100 \%$ and $e+\mathrm{Au} 0-10 \%$ at $20 \mathrm{GeV} \times 100 \mathrm{GeV}$ are shown in black, blue and red curves with the statistical uncertainty from an integrated luminosity of $10 \mathrm{fb}^{-1}$; (b) the ratio of the correlation function for $e+\mathrm{Au} 0-10 \%$ divided by $0-100 \%$.

If the ratio of correlation function from central divided by minimum bias is less than 1 , as suggested by Fig. 12(b) it could be an indication of saturation.

\section{Summary}

We have presented detailed studies on the determination of collision geometry in $e+\mathrm{A}$ collisions. Utilizing the DPMJET3 MC generator, we have found a correlation between the traveling length and the number of neutrons evaporated from the nuclear remnant. This neutron number distribution can be measured with a ZDC in the Augoing direction with the systematics under control. All the devices needed for this measurement have been included in the current EIC detector design. Therefore, it is very easy to acquire this measurement with little additional investment. Constraining collision geometry quantities like traveling length is very meaningful in the studies of cold nuclear medium effects. A demonstration of using this approach to make fine traveling length binning of performing nuclear medium energy loss studies and explore the signature of saturation physics has been provided. With the determination of collision geometry in these measurements, our understanding of nuclear structure can be constrained with higher precision.

We wish to thank Bo-Wen Xiao for helpful discussions and suggestions. This work was supported in part by the NSFC (11475068), the National Basic Research Program of China (2013CB837803), and the Basic Research Program of CCNU (CCNU13F026). E. C. A. and J. H. L. acknowledge support by the U.S. Department of Energy under Contract No. DE-AC0298CH10886.

\section{References}

1. A. Accardi, J. Albacete, M. Anselmino, N. Armesto, E. Aschenauer et al. (2012), 1212.1701
2. J.L. Albacete, N. Armesto, J.G. Milhano, C.A. Salgado, Phys.Rev. D80, 034031 (2009), 0902.1112

3. F. Dominguez, C. Marquet, B.W. Xiao, F. Yuan, Phys.Rev. D83, 105005 (2011), 1101.0715

4. A. Accardi, Phys.Rev. C76, 034902 (2007), 0706. 3227

5. W. Broniowski, W. Florkowski, Phys.Rev. C65, 024905 (2002), nucl-th/0110020

6. K. Aamodt et al. (ALICE Collaboration), Phys.Rev.Lett. 106, 032301 (2011), 1012.1657

7. B. Abelev et al. (STAR Collaboration), Phys.Rev. C79, 034909 (2009), 0808. 2041

8. B. Back et al. (PHOBOS Collaboration), Phys.Rev. C65, 061901 (2002), nucl-ex/0201005

9. A. Adare et al. (PHENIX Collaboration) (2013), 1310.4793

10. A. Toia (ALICE Collaboration) (2014), 1403.5143

11. S. Roesler, R. Engel, J. Ranft, pp. 1033-1038 (2000), hep-ph/0012252

12. M. Strikman, M. Tverskoii, M. Zhalov, Phys.Lett. B459, 37 (1999), nucl-th/9806099

13. S. White, M. Strikman (2010), 1003.2196

14. G. Piller, W. Ratzka, W. Weise, Z.Phys. A352, 427 (1995), hep-ph/9504407

15. M.L. Miller, K. Reygers, S.J. Sanders, P. Steinberg, Ann.Rev.Nucl.Part.Sci. 57, 205 (2007), nucl-ex/0701025

16. H.W. Bertini, Phys.Rev. 131, 1801 (1963)

17. V. Weisskopf, Phys.Rev. 52, 295 (1937)

18. A. Ferrari, P. Sala, J. Ranft, S. Roesler, Z.Phys. C70, 413 (1996), nucl-th/9509039

19. R. Engel, J. Ranft, S. Roesler, Phys.Rev. D55, 6957 (1997), hep-ph/9610281

20. R. Engel, J. Ranft, Phys.Rev. D54, 4244 (1996), hep-ph/9509373

21. K. Hanssgen, J. Ranft, Comput.Phys.Commun. 39, 37 (1986)

22. A. Ferrari, P.R. Sala, A. Fasso, J. Ranft (2005)

23. N. Akopov, L. Grigoryan, Z. Akopov, Eur.Phys.J. C44, 219 (2005), hep-ph/0409359

24. C.A. Salgado, U.A. Wiedemann, Phys.Rev. D68, 014008 (2003), hep-ph/0302184

25. N.B. Chang, W.T. Deng, X.N. Wang, Phys.Rev. C89, 034911 (2014), 1401.5109 
26. R. Charity, M. McMahan, G. Wozniak, R. McDonald, L. Moretto et al., Nucl.Phys. A483, 371 (1988)

27. J. Bondorf, A. Botvina, A. Ilinov, I. Mishustin, K. Sneppen, Phys.Rept. 257, 133 (1995)

28. S.Y. Shmakov, V. Uzhinsky, A. Zadorozhnyi, Comput. Phys. Commun. (1988)

29. G. Piller, W. Weise, Phys.Rept. 330, 1 (2000), hep-ph/9908230

30. M. Adams et al. (E665 Collaboration), Phys.Rev.Lett. 74, 5198 (1995)

31. C. Adler, A. Denisov, E. Garcia, M.J. Murray, H. Strobele et al., Nucl.Instrum.Meth. A470, 488 (2001), nucl-ex/0008005

32. A. Airapetian et al. (HERMES Collaboration), Phys.Lett. B577, 37 (2003), hep-ex/0307023

33. A. Airapetian et al. (HERMES Collaboration), Nucl.Phys. B780, 1 (2007), 0704.3270

34. A. Airapetian et al. (HERMES Collaboration), Eur.Phys.J. A47, 113 (2011), 1107.3496

35. L. Zheng, E. Aschenauer, J. Lee, B.W. Xiao, Phys.Rev. D89, 074037 (2014), 1403.2413 